\title{
Mejora de los indicadores biológicos de exposición al mercurio en trabajadores de una refinería de oro
}

\author{
Improvement of workers' biological indicators exposed to mercury at a gold refinery
}

\author{
Augusto V. Ramírez ${ }^{1,2}$ \\ American College of Occupational and Environmental Medicine. \\ ${ }^{2}$ Dirección Salud Ocupacional Clínica Los Fresnos.
}

\begin{abstract}
Resumen
Introducción: La exposición al mercurio asociada a malas prácticas de higiene laboral favorece el desarrollo de la intoxicación ocupacional denominada mercurialismo. Para verificar el impacto que la mejoría de los niveles ambientales de mercurio tendría en trabajadores de la refinería en una empresa aurífera peruana, se elaboró un programa de reingeniería para el control de la exposición que abarca aspectos de salud e higiene laboral y participación activa de la jefatura operativa. Objetivos: Estudiar en trabajadores de una refinería aurífera las variaciones de indicadores de exposición a mercurio al mejorar la calidad del ambiental laboral. Diseño: Estudio prospectivo y descriptivo. Lugar de estudio: Servicio de Salud Ocupacional de una empresa minera aurífera en el Perú. Participantes: Trabajadores de la refinería de oro. Intervenciones: Se modificó la metodología del examen médico y de la evaluación del ambiente laboral de 100 trabajadores de la refinería de oro, durante el periodo 2003-2007, además de mejorar las condiciones de trabajo. Luego, se cuantificó semestralmente los límites ambientales del tóxico y los indicadores de exposición. Se analizó estadísticamente la variación de los valores medios de los indicadores biológicos respecto a la variación del tóxico en el ambiente de trabajo. Principales medidas de resultados: Mejora de los indicadores ambientales y biológicos. Resultados: Los valores medios de los indicadores ambientales mejoraron cada año, al igual que los indicadores biológicos del universo estudiado. El mercurio ambiental en 2003 fue 6,3 $\mathrm{mg} / \mathrm{m}^{3} \mathrm{y}$, en 2 007, 0,03, valor de $\mathrm{p}<0,001$. El indicador mercurio urinario en 2003 fue 11,9 ug/L y, en 2007, 2,3 con $p<0,01$. Conclusiones: En el grupo estudiado, la disminución del tóxico en el ambiente de labor determinó la mejora significativa de los indicadores biológicos de exposición al mercurio en el trabajador.
\end{abstract}

Palabras clave: Intoxicación por mercurio; condiciones de trabajo; exposición profesional; ambiente de trabajo.

\section{Abstract}

Background: Occupational exposure to mercury is associated to mercurialism, a characteristic occupational poisoning. To verify impact of environmental mercury levels improvement would have in gold's refinery workers of a Peruvian auriferous company, we carried out a reengineering program for exposure control. This program included health and hygiene occupational aspects and operation's management active participation. Objective: To study in gold refinery workers variations in indicators of exposure to mercury with environment labor quality improvement. Design: Prospective and descriptive study. Place of study: Occupational Health Service in a Peruvian mining company. Participants: Workers of gold refinery. Interventions: Modification of physician examination methodology, hygienic evaluation and improved conditions of the labor environment. Every six months we quantified the environmental limits of both toxic and biological exposure indicators. Then variation of exposure biological indices versus variation of environmental limits was analyzed. Main outcome measures: Environmental and biological indicators. Results: Environmental indicators values improved every year, as well as workers biological indicators. Environmental mercury decreased from $6.3 \mathrm{mg} / \mathrm{m3}$ in 2003 to 0.03 at the end of 2007, p< 0.001 . Urinary mercury decreased from $11.9 \mathrm{ug} / \mathrm{L}$ in 2003 to 2.3 in 2007, p < 0.01. Conclusions: In the group studied workers' toxic levels decreased with improvement of the environment indicator.

Key words: Poisoning by mercury; working conditions; occupational exposure; work environment.

An Fac med. 2011;72(3):177-82

\section{INTRODUCCIÓN}

Los principales riesgos de enfermedad ocupacional en minería aurífera por lixiviación son los químicos y de ellos el mercurio es uno de los mayores. El mercurio $(\mathrm{Hg})$ es un metal blanco-plateado, muy tóxico, el único en estado líquido a $0^{\circ} \mathrm{C}$, muy denso y poco compresible, de tensión superficial muy alta y débil reacción calorífica; posee gran capaci- dad de amalgamar a casi todos los metales. Se evapora a $13^{\circ} \mathrm{C}$ y encontramos trazas de él en cualquier producto que se analice. No es esencial para proceso biológico alguno, pero se acumula en la mayoría de los seres vivos ${ }^{(1)}$. En el medioambiente, aparte del estado natural, existe un importante aporte antropogénico proveniente de su metalurgia, de sus aplicaciones en industrias diversas y de las aguas residuales de las ciudades, pues se calcula que cada año mil toneladas son liberadas de las redes de alcantarillado a la superficie ${ }^{(2-4)}$.

La toxicidad del mercurio es conocida desde antiguo por Hipócrates, Plinio y Galeno; mas, las primeras notificaciones de sus efectos tóxicos como riesgo laboral, son las de Ellenberg, en 1473. Luego, Paracelso, en 1533, y Ramazzi- 
ni en De Morbis Artificium Diatriba, c. 1700, describen el cuadro clínico de intoxicación ocupacional. Paracelso sostiene que "Solo la dosis determina los efectos de un tóxico"; ergo, a mayor dosis mayor efecto. Igual, en salud ocupacional hoy es un axioma que a mayor cantidad de tóxico en el ambiente de labor, mayor su concentración en el organismo del trabajador ${ }^{(5)}$.

La exposición a mercurio asociada a malas prácticas de higiene laboral favorece el desarrollo de intoxicación, que se manifiesta por un cuadro clínico de características propias denominado mercurialismo ${ }^{(1,5-6)}$. En medicina del trabajo no existe unanimidad en cuanto al umbral de toxicidad humana, pero la investigación actual en salud lo fija entre 50 y 160 ug/día ${ }^{(7-9)}$.

Hacia la mitad del siglo pasado se establecieron los llamados niveles o límites permisibles, ambientales y sistémicos para el trabajo con tóxicos, determinando que si estas cantidades se incrementaban, el trabajador enfermaba. Para estos límites existen diferentes denominaciones, que dependen del país donde transcurre el trabajo. En América, usamos los valores de la American Conference of Governmental Industrial Hygienist $\left(\mathrm{ACGIH}^{\circledR}\right)$, cuya denominación es valores umbrales límite (threshold limit values - TLV ${ }^{\oplus}$ ) para magnitudes del tóxico en el ambiente de labor y límites de exposición biológica (biological exposure limits - $\mathrm{BEI}^{\circledR}$ ) en los fluidos del trabajador. También, se llama BEI a los metabolitos o a los productos de excreción de un tóxico ${ }^{(8-10)}$.

En toxicología ocupacional conocemos al mercurio como uno de los metales pesados más agresivos. En metalurgia del oro aparece como subproducto durante su refinación y es en esta fase del proceso donde se genera el mayor riesgo de intoxicación ocupacional. Las empresas mineras formales protegen a sus trabajadores técnica y adecuadamente, pero aún así, puede acaecer que por alguna eventualidad desmejoren los controles del ambiente laboral y el tóxico se incremente ${ }^{(1,5-7,10)}$.
Para verificar el impacto que la mejoría de los niveles ambientales de mercurio podría tener en los trabajadores de refinería en una empresa aurífera de la gran minería peruana, cuyos indicadores ambientales se habían mantenido sobre el TLV y los BEI en magnitudes que sin sobrepasarlo eran definidamente altas, se elaboró un programa para reingeniería del control específico de exposición a este tóxico, técnicamente formulado, y que concomitantemente comprendiera aspectos de salud e higiene laboral, comprometiendo además la participación activa de la jefatura operativa del área.

Luego del diagnóstico situacional realizado en 2002, se propuso a la Empresa realizar mejoras en el programa de vigilancia por exposición a mercurio, con miras a prevenir el riesgo de intoxicación ocupacional y, por tanto, prevenir daño a la salud en los trabajadores expuestos ${ }^{(11-14)}$.

\section{MÉTODOS}

Nuestro estudio prospectivo y descriptivo tuvo como objetivo determinar y medir la variación de los BEI de exposición a mercurio y de los valores ambientales del tóxico en trabajadores de una empresa minera aurífera durante el quinquenio 2003 a 2007, concomitante a un proceso de reingeniería en salud e higiene laboral que abarcaba las siguientes variables: ambiente de trabajo, salud e higiene ocupacional y conocimiento del tóxico por el trabajador.

El estudio incluyó a todos los trabajadores regulares y en planilla del área Procesos metalúrgicos en la refinería de oro de la empresa. Excluyó a los trabajadores que ingresaban eventual- mente al área para realizar trabajos de mantenimiento; a los trabajadores que por diversos motivos se ausentaban temporalmente por más de un mes, por ejemplo para capacitación; a los trabajadores que se retiraban definitivamente; y al personal nuevo que ingresaba a trabajar al área.

El universo al final del estudio fue de 100 trabajadores, con media de edad al inicio de 34 años y valores extremos entre 26 y 48 años.

Antes de la implementación del programa, los trabajadores fueron informados de los cambios que se llevarían a cabo, tanto en el examen médico cuanto en la toma de muestras biológicas y que semestralmente se les haría conocer el resultado de sus exámenes ${ }^{(15)}$.

En la evaluación, Salud Ocupacional estandarizó el examen clínico, la metodología de toma de muestras biológicas y seleccionó un laboratorio certificado en dosaje de metales pesados. Para realizar estas acciones diseñó un protocolo específico aprobado por el Ministerio Energía y Minas ${ }^{(11)}$. Este protocolo, en adición al examen clínico, usó el BEI mercurio urinario de ACGIH como parámetro de exposición para control biológico, complementado con los $\mathrm{BEI}$ creatinina en orina de 24 horas y proteínas totales y, cuando fue requerido, utilizó el marcador beta ${ }_{2}$ micro globulina. Semestralmente, se examinó a todo el universo. El análisis del mercurio en orina fue realizado por absorción atómica con horno de grafito ${ }^{(11)}$.

El valor de referencia (VR) para los indicadores biológicos en el lapso del estudio consta en la tabla 1.

Para valorar el mercurio en el ambiente de labor, Higiene Industrial utiliza los siguientes equipos:

Tabla 1. Valor de referencia para los indicadores biológicos utilizados.

$\begin{array}{cc}\text { Mercurio en orina (ACGIH) } & <40 \mathrm{ug} / \mathrm{L} \\ \text { Creatinina en orina } & 0,4 \text { a } 1,8 \mathrm{~g} / 24 \mathrm{~h} \\ \text { Proteínas totales en orina } & \text { Hasta } 140 \mathrm{mg} / 24 \mathrm{~h}\end{array}$


a. Muestreador pasivo SKC ${ }^{\circledR} \mathrm{PMI}$ serie 520 , validado con el procedimiento ID 140 OSHA, de uso individual y que básicamente es una bomba diseñada para toma de muestras personales, de buen resultado en captación de mercurio; consta de una carcasa reutilizable y una cápsula adsorbente restituible; reporta como peso promedio respecto al tiempo, TWA ${ }^{(16)}$.

b. Jerome ${ }^{\circledR} 431-X$ : equipo estacionario para muestreo ambiental basado en un sensor de membrana diseñado para detectar los gases de mercurio en el aire ambiental ${ }^{(17)}$.

En total, por año se toma 24 muestras en el área respiratoria del trabajador más 60 (5 x mes) muestras estacionarias. De los resultados, y para el análisis, en cada año se toma los valores medios. El análisis de mercurio ambiental se hace por absorción atómica sin llama ${ }^{(12)}$.

El estándar ambiental utilizado es el de ACGIH: $0,025 \mathrm{mg} / \mathrm{m} 3 / 8$ horas $/ 5$ días semana ${ }^{(8)}$, al que se corrige para 48 horas/semana ${ }^{(8,9)}$.

Como parte de la implementación del programa y por recomendación de Salud e Higiene Ocupacional, Operaciones Metalúrgicas de la empresa ${ }^{(12)}$ ejecutó las siguientes acciones de mejora del medio ambiente laboral:

\section{a. Básicas}

- Monitoreo de vapor de mercurio por etapas de trabajo, adicional al muestreo de turno completo.

- Contratación de personal a dedicación exclusiva para la limpieza de los ambientes de labor.

- Implementación de ropa de trabajo adecuada y moderna.

- Restricción del acceso indiscriminado a las áreas externas de retortas y filtros prensa, áreas gravitantes en liberación del tóxico.

- Limpieza meticulosa del área con $\mathrm{HgX}$ en polvo, un neutralizante químico del $\mathrm{Hg}$.

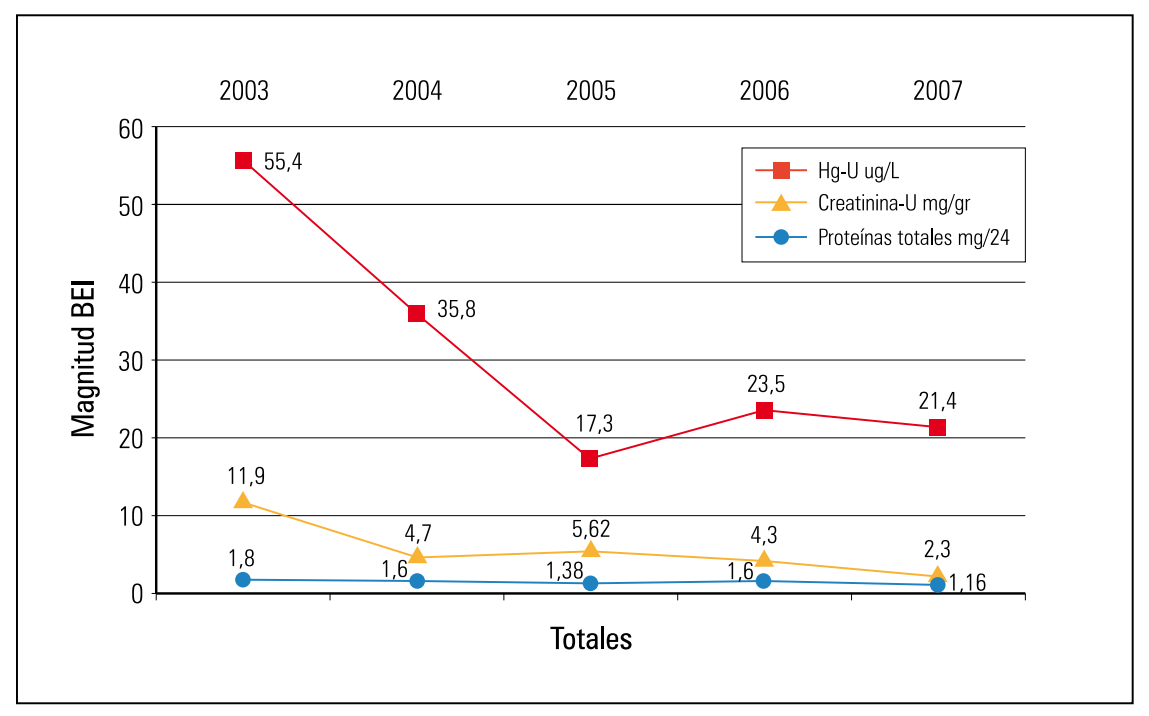

Figura 1. Media anual comparativa BEls.

- Capacitación en el conocimiento del tóxico a los trabajadores involucrados.

- Cambio de metodología en la toma de muestra de orina, que pasó a ser responsabilidad de salud ocupacional, a fin de ser realizada técnicamente y fuera del área de operaciones ${ }^{(11)}$.

\section{b. Complejas}

- Mejoramiento de los sistemas de ventilación y de los equipos de la refinería.
- Modificación del separador de mercurio.

- Mejoramiento del tiempo de enfriamiento en las retortas.

- Mejora en el manejo del precipitado.

- Implementación de ventiladores portátiles adicionales en las áreas de labor, en la eventualidad que los fijos se sobrecargaran.

A fin de mejorar el conocimiento por los trabajadores de los riesgos del tóxico con que laboran, la empresa au-

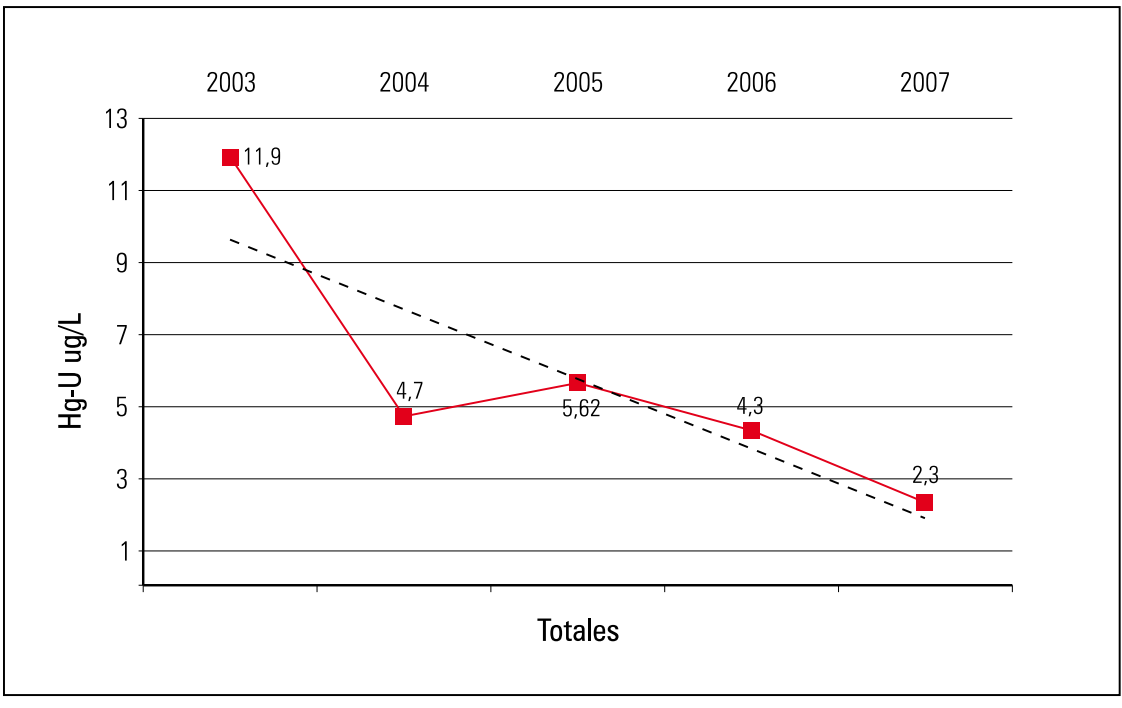

Figura 2. Media anual de mercurio urinario. 


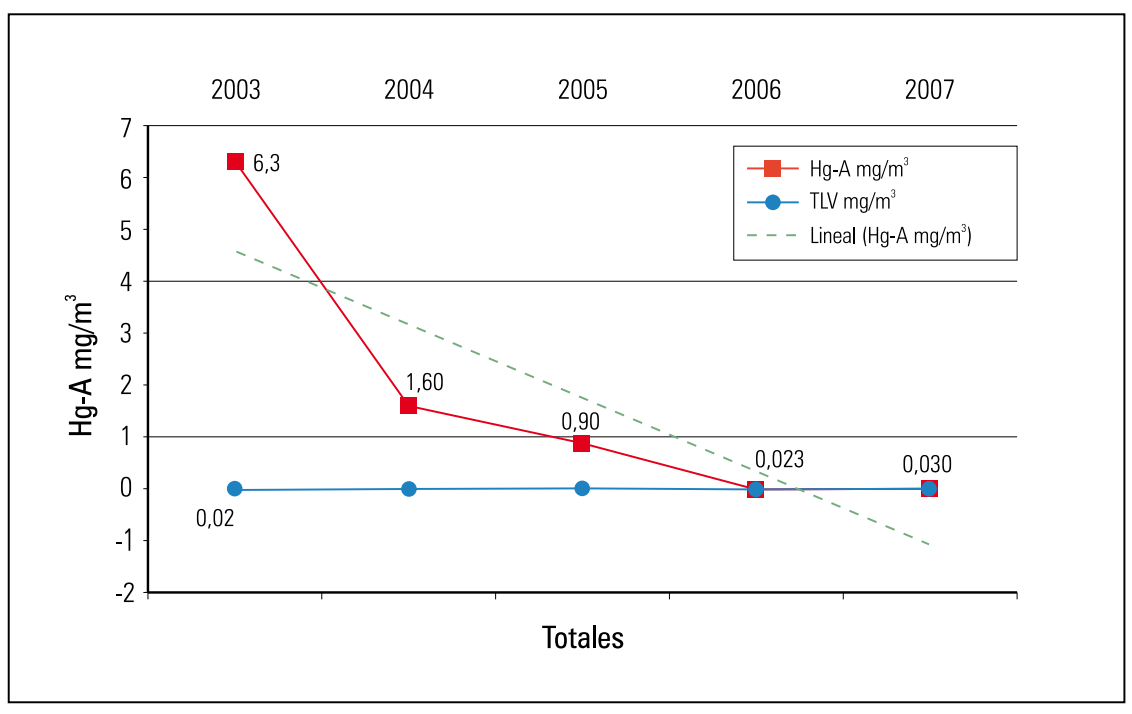

Figura 3. Media anual de mercurio ambiental (ambiente y personal), procesos $2003-2007$.

torizó a salud e higiene ocupacional a realizar cursos, charlas y talleres para el grupo de refinería fuera de los horarios y ambientes de labor.

La principal limitante de nuestra investigación fue el corto lapso de estudio, pues por consideraciones ajenas a salud e higiene ocupacional solo pudimos evaluar durante cinco años a esta población.

Otra limitación es no reportar aquí la mejoría del cuadro clínico, debido a que rebasaría el objetivo del estudio y lo extendería; sin embargo, se preparó y reportó resultados generales a la empresa e individualmente a los trabajadores.

Los TLV ambientales de mercurio y los BEI en los trabajadores fueron evaluados en forma estadística y por separado en cada año del quinquenio. Obtuvimos media aritmética, desviación estándar y rango. En 2003 se estableció los valores iniciales de los BEI (Hg-U, creatinina y proteínas totales) y

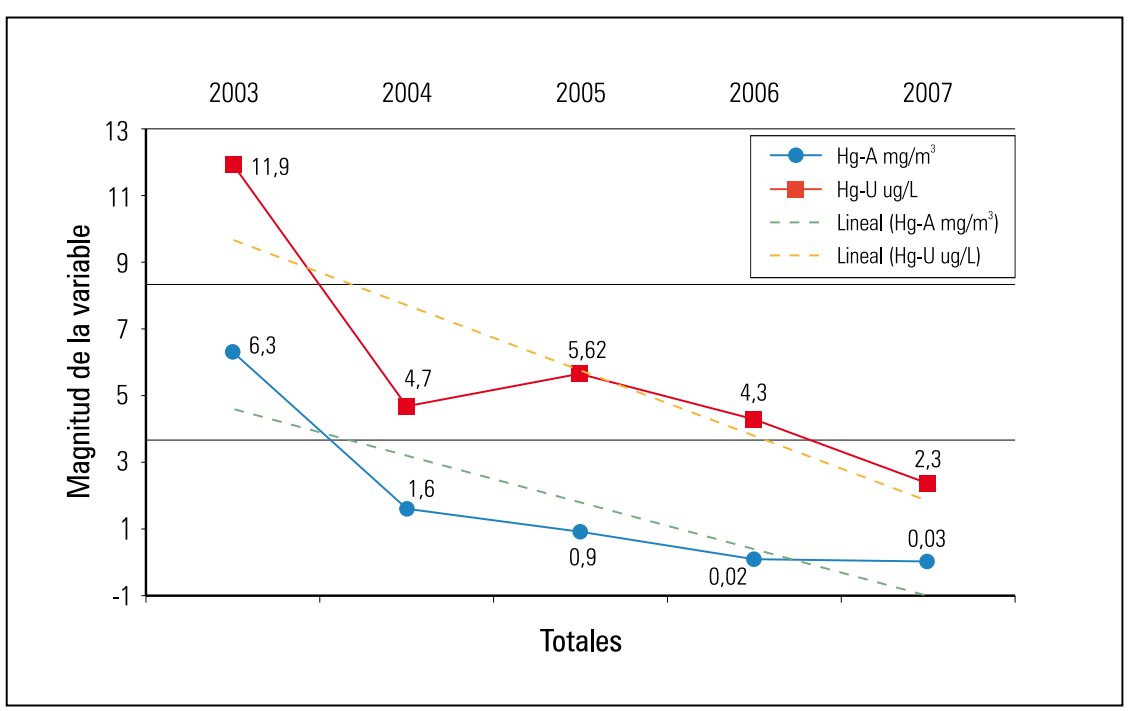

Figura 4. Comparativo media anual Hg-ambiente versus Hg-urinario.

del TLV ambiental de mercurio en el área de labor y se les acompañó anualmente durante el estudio, que concluyó en 2007.

Se comparó los valores medios anuales entre los TLV y los BEI, estableciéndose el valor de $t$ por la prueba $t$ de Fisher y el significado estadístico de las diferencias mediante cálculo de la probabilidad $p^{(18)}$. Para procesar los datos, usamos el programa Excel $^{\oplus}$.

\section{RESULTADOS}

La figura 1 muestra la evolución favorable de los BEI estudiados (mercurio urinario, creatinina urinaria y proteínas totales). La figura 2 expresa la media anual y la tendencia de evolución del mercurio urinario. En la figura 3 se presenta la evolución en el quinquenio de la media anual del $\mathrm{Hg}-\mathrm{U}$ (media de la medición personal más la medición ambiental). La figura 4 compara la evolución del mercurio ambiente versus mercurio urinario.

Los resultados puntuales hallados en los BEI (mercurio urinario, creatinina urinaria y proteínas totales) se muestra en detalle en tablas. La tabla 2 presenta los resultados generales del estudio, consignado los valores medios, desviación estándar y rango de los BEI para mercurio en el organismo de los trabajadores y del TLV ambiental. La tabla 3 muestra el resultado del valor medio anual de mercurio ambiental en las áreas de labor durante el lapso de estudio. La tabla 4 reúne los resultados del valor medio anual de los indicadores biológicos de exposición al tóxico durante el quinquenio. La tabla 5 muestra la comparación estadística de los valores ambientales y biológicos entre los años 2003 y 2007, estableciendo el valor de $p$ para cada uno de ellos.

\section{DISCUSIÓN}

En nuestro país no existen publicaciones acerca de estudios para el tóxico mercurio. Lo publicado en el exterior 
Tabla 2. Resultados generales por indicador por año *.

\begin{tabular}{ccccc} 
Año** & $\begin{array}{c}\text { Mercurio ambiente } \mathrm{mg} / \mathrm{m}^{3} \\
\mathrm{n}: 84\end{array}$ & $\begin{array}{c}\text { Mercurio urinario ug/ L } \\
\mathrm{n}: 200\end{array}$ & $\begin{array}{c}\text { Creatinina urinaria g/24 h } \\
\mathrm{n}: 200\end{array}$ & $\begin{array}{c}\text { Proteínas totales en orina mg/24 h } \\
\mathrm{n}: 200\end{array}$ \\
2003 & $6,3 \pm 3,75(47,2-0,8)$ & $11,9 \pm 8,3(14,2-8,4)$ & $1,8 \pm 1,12(2,0-0,4)$ & $55,4 \pm 24,7(94-46)$ \\
2004 & $1,6 \pm 0,49(1,9-0,8)$ & $4,7 \pm 0,49(6,2-3,6)$ & $1,6 \pm 0,5(2,1-0,6)$ & $35,8 \pm 14,8(62-30)$ \\
2005 & $0,9 \pm 0,15(3,5-2,1)$ & $5,62 \pm 0,97(9,4-3,9)$ & $1,38 \pm 0,28(1,81-0,52)$ & $17,3 \pm 6,14(38-16.8)$ \\
2006 & $0,02 \pm 0,009(0,045-0,0012)$ & $4,3 \pm 1,46(7,1-3,9)$ & $1,6 \pm 0,8(1,87-0,46)$ & $23,5 \pm 1,13(29-24)$ \\
2007 & $0,03 \pm 0,007(0,047-0,0027)$ & $2,3 \pm 0,21(4,6-0,9)$ & $1,16 \pm 0,21(1,59-0,57)$ & $21,4 \pm 0,7(23-20)$ \\
\hline
\end{tabular}

* Media \pm desviación estándar (rango). ** Número de muestras por año.

Tabla 3. Valores medios anuales de mercurio ambiental, $2003-2007$.

\begin{tabular}{cccccc} 
n: $84 /$ año & 2003 & 2004 & 2005 & 2006 & 2007 \\
$\mathrm{Hg}-\mathrm{A} \mathrm{mg} / \mathrm{m}^{3}$ & 6,3 & 1,6 & 0,9 & 0,02 & 0,03 \\
\hline
\end{tabular}

Tabla 4. Valores medios anuales de los indicadores biológicos de exposición. N trabajadores: 100.

\begin{tabular}{cccc} 
Año & $\begin{array}{c}\mathrm{Hg}-\mathrm{U} \\
\mathrm{ug} / \mathrm{L}\end{array}$ & $\begin{array}{c}\text { Creatinina-U } \\
\mathrm{mg} / \mathrm{g}\end{array}$ & $\begin{array}{c}\text { Proteínas totales } \\
\mathrm{mg} / 24 \mathrm{~h}\end{array}$ \\
2003 & 11,9 & 1,8 & 55,4 \\
2004 & 4,7 & 1,6 & 35,8 \\
2005 & 5,62 & 1,38 & 17,3 \\
2006 & 4,3 & 1,6 & 23,5 \\
2007 & 2,3 & 1,16 & 21,4 \\
\hline
\end{tabular}

Tabla 5. Comparación TLV mercurio ambiental y de los BEls, 2003 vs. 2007.

\begin{tabular}{lcccc} 
& & 2003 & 2007 & Valor de $p$ \\
\hline Hg ambiental & & & & \\
BEl & $H g-A ~ m g / m^{3}$ & 6,3 & 0,03 & $<0,001$ \\
\hline & & & & \\
\hline & $H g-U$ ug/L & 11,9 & 2,3 & $<0,01$ \\
& Creatinina $\mathrm{mg} / \mathrm{gr}$. & 1,8 & 1,16 & $<0,1$ \\
& Proteínas totales mg/24 h & 55,4 & 21,4 & $<0,1$ \\
\hline
\end{tabular}

es abundante, aunque data de antes del año $2000\left({ }^{2,19-23)} \mathrm{y}\right.$ unos pocos son posteriores ${ }^{(24)}$.

Nuestra aproximación enfoca el problema desde el punto de vista de la salud ocupacional como dependiente de las condiciones de higiene del lugar de trabajo. Para realizarla, valoramos los índices de exposición en el trabajador y los contrastamos con la magnitud del tóxico en el ambiente de labor. En ese sentido, desarrollamos un protocolo que permitiese un mejor control de salud ocupacional en el grupo y, que ade- más de los exámenes ocupacionales por la exposición, vigilase que la muestra biológica se tomara, manejara y examinara en condiciones técnicas adecuadas, con el fin de representar fehacientemente la cantidad del xenobiótico en el organismo. Además, salud e higiene ocupacional imparten al trabajador conocimientos básicos del tóxico con el que labora, a fin de que, consciente del riesgo, se proteja.

Como resultado las acciones de higiene industrial, se logró revertir las concentraciones de vapor de mercu- rio en los ambientes de trabajo, hasta alcanzar magnitudes similares o por debajo de los límites permisibles adoptados por la empresa, en concordancia con la norma internacional $(<0,020$ $\mathrm{mg} / \mathrm{m} 3 / 48$ horas semana) ${ }^{(8)}$; así, los valores medios anuales, de $6,3 \mathrm{mg} / \mathrm{m}^{3}$ en 2003 cayeron a $0,03 \mathrm{mg} / \mathrm{m}^{3}$ para 2007 , $\mathrm{p}<0,001$.

En salud ocupacional, los valores medios de los indicadores de exposición en el lapso 2003 a 2007 establecieron que el mercurio en orina de 24 horas, nuestro principal indicador de exposición, disminuyó su valor progresiva y sustancialmente desde los iniciales 11,9 ug/L de 2003 a 2,3 ug/L en 2007 (VR durante todo el periodo, $\mathrm{Hg}-\mathrm{U}<$ $40 \mathrm{ug} / \mathrm{L}$ ); la creatinina, de $1,8 \mathrm{mg} / \mathrm{g}$ en 2003 disminuyó a 1,16 en 2007. Igual sucedió con las proteínas urinarias totales, que disminuyeron de 55,4 mg/24 h en 2003 a 21,4 en 2007. Estos resultados concuerdan con estudios similares realizados en España por Español (2) y por Cabanillas ${ }^{(20)}$, en EE UU por Bertram ${ }^{(21)}$, Berlim ${ }^{(22)}$ y Lapare ${ }^{(24)}$, y a los de Leidel ${ }^{(23)}$, quien en una investigación para la NIOSH estableció que el nivel de mercurio urinario depende de su concentración en el ambiente.

En el análisis estadístico, los resultados mostraron que los valores medios anuales de Hg-U 2003 a 2007 disminuyeron significativamente a nivel de $p<0,01$, lo que concordó con la disminución del mercurio en el ambiente durante el mismo periodo, $\mathrm{p}<0,001$. Los otros indicadores también disminuyeron, pero con menor significancia: creatinina $\mathrm{p}<0,1$ y proteínas totales $\mathrm{p}$ $<0,1$; indirectamente evidencian que, 
a pesar de disminuir la carga corporal de mercurio, los indicadores de impacto orgánico disminuyeron a menor velocidad, hallazgo similar a lo encontrado por Lavejoy, en $1973^{(19)}$, y por Español, en $1990^{\text {(2). }}$

Nuestra investigación concluye que, el control del riesgo químico por mercurio, técnicamente concebido con acciones conjuntas e integradas de salud, higiene ocupacional y operaciones, deviene en mejoría de los indicadores biológicos de exposición lo que, sin redundar, está acorde con el criterio de Salud Ocupacional: cuando se mejora los niveles ambientales del tóxico, los indicadores biológicos en el trabajador disminuyen.

\section{REFERENCIAS BIBLIOGRÁFICAS}

1. Zenz C, ed. Occupational medicine: principles and practical applications. 2a ed. Chicago: Mosby; 1988.

2. Español CS. Estudio sobre correlación de valores de mercurio en ambiente y fluidos biológicos con la aparición de sintomatología clínica en trabajadores de Minas de Almadén y Arrayanes. España: Ed. Minas de Almadén; 1990.

3. Parmeggiani L, ed. Encyclopedia of Occupational Health and Safety. 3th Ed. Geneva: International Labor Office; 1989.

4. Organización Mundial de la Salud. El Mercurio en el Sector de la Salud. Departamento de Salud Pública y Medio Ambiente Agua, Saneamiento y Salud. Ginebra. OMS; 2004.

5. Ellemhorn MJ. Medical Toxicology. 2nd Edition. Baltimore: Williams Wilkins; 1996.

6. Casarett and Doull's. Toxicology. The Basic Science of Poison. 6th Edition. Barcelona: Mc Millan; 2001.
7. Friberg L, Nordberg GF, Vouk VB, eds. Handbook of toxicology of metals. Amsterdam: Ed. Elsevier; 1979.

8. ACGIH, TLVs. BEls. Threshold limits values for chemical substances and physical agents. Biological exposure indices. Cincinnati: ACGIH; 2008.

9. U.S. Department of Labour. Occupational Safety and Health Administration. Air Contaminants-Permissible Exposure Limits (Title 29 Code of Federal Regulations Part 1910.1000) USA. OSHA 3112; 1989.

10. Ramírez AV. Biomarcadores en monitoreo de exposición a metales pesados en metalurgia. An Fac med. 2006;67(1):49-58.

11. Ramirez AV, Fiestas SE. Protocolo de Vigilancia en Salud Ocupacional. Riesgo químico mercurio. Lima. Pacifico Salud, Medica; 2005. Disponible en: http://intranet2.minem.gob.pe/web/archivos/ dgm/publicaciones/expedientes/yanacocha/ justificacion/4.2R3\%20Protocolo\%20de\%20Vigilancia $\% 20$ en $\% 20$ Salud $\% 20$ Ocupacional\%20de $\% 20$ Riesgo\%20Qu\%C3\%ADmico-Mercurio-2005.pdf. Visita: noviembre 2010.

12. Bancayán L, Ramírez AV. El Control del Contaminante Mercurio, en la Producción de Oro. En: Actas III Seminario Internacional de Salud Ocupacional en Operaciones Mineras. Lima. ISEM; octubre 2008.

13. United Nations Environment Program. Maximum Allowable Concentrations and Tentative Safe Exposure Levels of Harmful Substances in the Environmental Media (Hygienic Standards Officially Approved). Centre of International Projects, USSR. GKNT; 1984

14. NIOSH U.S, National Institute for Occupational Safety and Health: TLV- CS Committee, Operations Manual of the Threshold Limit Value for Chemical Substances. Disponible en www.acgih.org/TLV/ OPSManual .pdf. Visita: enero 2011.

15. Asociación Médica Mundial. Declaración de Helsinki. Principios éticos aplicables a las investigaciones médicas que involucran seres humanos. 18 Asamblea General de Helsinki, junio 1964. Última Revisión: 52 Asamblea General, Edimburgo, Escocia; octubre 2000.

16. Airlite USA. Personal Modular Impactor PMI: USA SKC AirLite; 2010 Disponible en: http://www.skcinc. com/PDB.asp. Visita: enero 2011.

17. Quiminet. Jerome 431-X Muestreador de gases Industriales. Quiminet USA; 2010. Disponible en:
http://www.quiminet.com/pro/Analizador\%2Bcont nuo\%2Bde\%2Bgases.htm. Visita: enero 2011.

18. Reynaga O J. Epidemiologia IV: Diseños de investigación para comprobación de hipótesis epidemiológicas. México DF. Centro Panamericano de Ecología Humana y Salud. OPS; 1985.

19. Lovejoy HB, Bell ZB Jr. Mercury exposure evolution and their correlation with urine mercury excretions. J Occup Med. 1973;15:1964.

20. Cabanillas MJL. Evaluación epidemiológica de un programa preventivo en Salud laboral. Mapfre Medicina. 1998;9:257-64.

21. Bertram D. La relación dosis-respuesta tras la exposición a compuestos de alquil-mercurio. Michigan University; 1976. En: Español CS. Estudio sobre correlación de valores de mercurio en ambiente y fluidos biológicos con la aparición de sintomatología clínica en trabajadores de Minas de Almadén y Arrayanes. España: Ed. Minas de Almadén; 1990.

22. Berlim M, Dose-response relations and diagnostic indices of mercury concentrations in critical organs upon exposure to mercury and mercurial's. En: Nordberg GF (Ed). Effects and dose-response relations of toxic metals. Amsterdam: Elsevier; 1976:235-45.

23. Leidel NA, Bush KA, Crouse WE. Exposure measurement level and occupational environmental variability. Mercury. US National Technical Information Service. Springfield VA; DHEW (NIOSH); 1975 76-131.

24. Lapare S, Brodeur J, Tardif R. Contribution to toxic kinetic modeling to the adjustment of exposure limits: mercury. Am Ind Hyg Assoc J. 2003;64(1):17-23.

Financiamiento: Ninguno.

Conflictos de interés: Ninguno.

\section{Correspondencia:}

Augusto V. Ramirez, MD

American College of Occupational and Environmental Medicine

Dirección Salud Ocupacional Clínica Los Fresnos

Jr. Los Nogales 149, Cajamarca, Perú.

Teléfono: 51076364046 Ext. 244

Correo electrónico: augustovram@yahoo.es 